\title{
Research of barometric altitude measurement technology
}

\author{
Ye ZHAN ${ }^{1, a}$, Li Min CHANG ${ }^{1}$ and Jun $\mathrm{LI}^{1}$ \\ ${ }^{1}$ Aircraft control Department, Air Force Aviation University, Changchun, China
}

\begin{abstract}
The atmospheric pressure measurement is closed related to the aircraft flight and it is the key factor directly affecting the aviation flight safety. With the development of aircraft, the barometric altitude measurement technology is more and more prominent and important. According to the barometric altitude measurement principle and the general barometric altitude measurement methods, four kinds of measurement methods of bellows, vibration cylinder, silicon resonant and silicon piezoresistance are analyzed with the principle and compared from the application.
\end{abstract}

\section{Introduction}

In the aerospace field, the atmospheric pressure measurement is closed related to the aircraft flight. From the aviation perspective, the height of aircraft is indicated by the pressure altimeter in the flight. The pressure altimeter is the instrument indicating the flight altitude through the atmospheric pressure. Measuring and choosing the flight altitude correctly is greatly important for playing the aircraft performance sufficiently, reducing the fuel consumption, saving the flight time and ensuring the flight safety. The pressure altimeter is installed on all modern kinds of aircraft. However, this kind of altimeter is with a certain hidden trouble because of the natural principle characteristics used by barometric altimeter.

\section{Barometric altitude measuring principle}

Assuming that the atmosphere is relatively still to the earth, there is no horizontal and vertical movement of atmosphere. Then, a thin layer of air column is got at any height, which is shown in Figure 1. The air column height is $d H$ and the cross section area is $\mathrm{dF}$. The air density is $\rho$ and the downward orientation is the positive direction. And the static equilibrium condition for the air column is as followed.

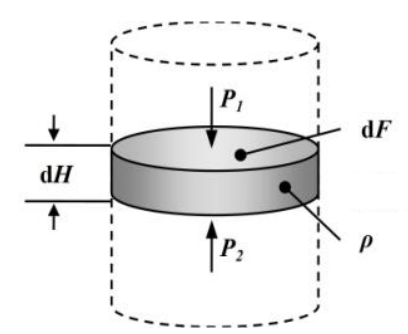

Figure 1.Force analysis of thin layer air column

$$
d P \cdot d F+\rho g \cdot d H \cdot d F=0 \quad d P=P_{1}+P_{2}
$$

Due to $P=\rho g R T$, the equation can be transformed to

$$
\frac{d P}{P}=-\frac{1}{R T} d H
$$

Due to the changing of atmospheric temperature vertical change rate $\tau$ with the change of time and place, the temperature at one height can be sensed accurately if the average temperature vertical change rate $\tau=-6.5 \mathrm{~K} / \mathrm{km}$ of troposphere is used in practical application. Therefore, the standard barometric altitude formula is taken place by the Laplace barometric altitude formula on approximation.

$T$ is taken place by $T_{\mathrm{m}}$ of temperature arithmetic mean and the equation (2) is integrated as follow.

$$
\int_{P_{b}}^{P_{H}} \frac{d P}{P}=\int_{H_{b}}^{H} \frac{1}{R T_{m}} d H
$$

And the following equation can be got.

$$
H=R T_{m} \ln \frac{P_{b}}{P_{H}}
$$

The algorithms are different in different levels. In the troposphere below $11000 \mathrm{~m}$, Tm of average temperature is calculated by the following equation (4).

$$
T_{m}=\frac{T_{b}+T_{H}}{2}=\frac{T_{b}+\left(T_{b}+\tau H\right)}{2}=T_{b}+\frac{\tau H}{2}
$$

In the flight altitude range of $11000 \sim 20000 \mathrm{~m}$, the average temperature $T_{\mathrm{m}}$ is calculated by the following equation (5).

$$
T_{m}=\frac{\frac{T_{b}+T_{11}}{2} H_{11}+T_{11}\left(H-H_{11}\right)}{H}=\frac{\left(T_{b}-T_{H}\right) H_{11}}{2 H}+T_{11}
$$

The altitude exist the one-to-one relationship with the barometric pressure. It has been found that the altitude calculated by the approximate formula is more close to the actual barometric altitude if the datum temperature $\left(T_{\mathrm{b}}\right)$ and the high altitude temperature $\left(T_{\mathrm{H}}\right.$ and $\left.T_{11}\right)$ used

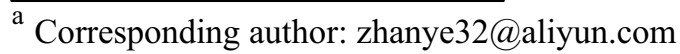


the actual measured values in actual atmosphere. Therefore, the barometric altitude approximate formula is used in some air data system.

\section{Barometric altitude measuring technology}

\subsection{Bellows barometric altimeter}

The bellows barometric altimeter is designed according to the standard atmospheric conditions. The air static pressure outside will change when the barometric altitude changes. And the pressure sensed by the vacuum bellows changes so the bellows center will output the tiny linear displacement. The displacement is transmitted and magnified through the connecting gear and the indicating section will indicate the barometric altitude after changing. Its core component is the vacuum bellows, which is shown in Figure 2.

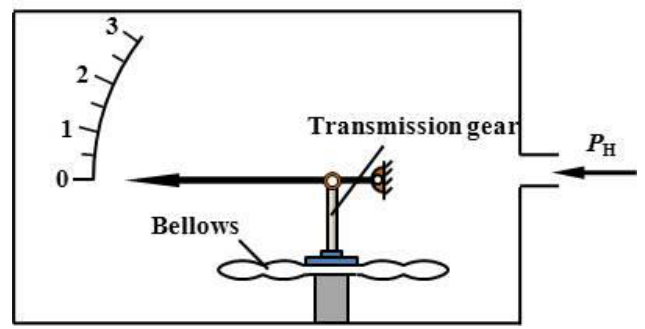

Figure 2.Principle diagram of barometric altimeter

The sensing component of bellows barometric altimeter is the vacuum bellows. The bellows inside is vacuum and the outside senses the atmosphere static pressure. Its pressure-displacement characteristic curve is similar with the atmospheric pressure-altitude characteristic curve. It is shown in Figure 3.

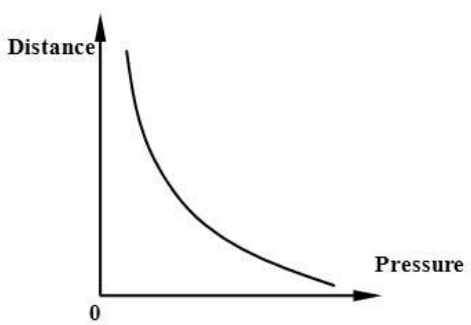

Figure 3. Characteristic curve of vacuum bellows

The early atmosphere data measurement was done though the bellows instrument. Although the structure of bellows instrument is simple and the operation is reliable, the precision is low.

\subsection{Vibration cylinder pressure altimeter}

The vibration cylinder pressure sensor component is mainly composed of the static pressure sensor and the total pressure sensor. The structure of vibration cylinder sensor is shown in Figure 4. It consists of the vibration cylinder, the single coil excitement, the vibration pickup coil, the foundation bed and the outer protective barrel.
The vibration cylinder is the core component, whose top is closed as free and the other end is fixed on the foundation bed. The space is a vacuum between the vibration cylinder and the protective barrel, which is as a reference for the measured pressure. The pressure sensed by the vibration cylinder is the absolute pressure when the measured pressure went into the space between vibration cylinder and coil.

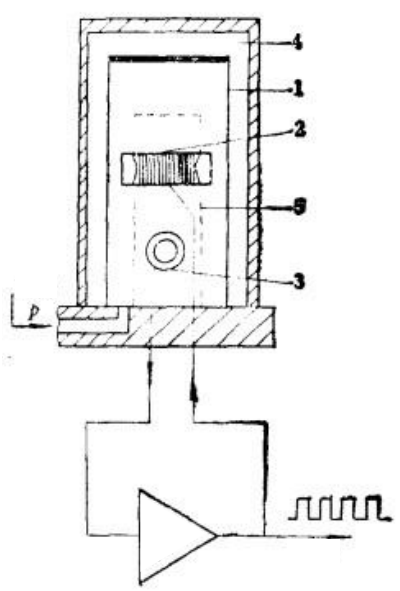

Figure 4.Principle block diagram of vibration cylinder sensor

The vibration cylinder pressure sensor component is the sensitive part of altimeter. It receives the static pressure and the total pressure from the pitot tube of aircraft and the pressure are converted into the frequency signal which is with a one-to-one correspondence relationship with pressure.

When the power is not on, the vibration cylinder is the stationary state. When turning on the DC power supply, the natural noise of amplifier products a weak random pulse in the excitation coil. The pulse signal make the magnetic field change through the excitation coil, which forming the pulse power. The pulse power cause the deformation of vibration cylinder wall and the vibration cylinder vibrates with the resonance frequency of low amplitude.

When the measured pressure is zero, the natural vibration frequency of vibration cylinder is $f_{0}$. When the measured pressure is changed, the vibration cylinder stiffness change and its resonant frequency also change accordingly. The vibration pick-up coil sense the vibration frequency changing with pressure on one hand and keep the vibration cylinder maintain the persistent oscillation through the positive feedback on the other hand. And the vibration frequency is $f=f_{0} \sqrt{1+A P}$. $P$ is the measured pressure, $A$ is the vibration cylinder constant. Due to the amplitude limiting in the circuit, the maximum deformation of vibration cylinder is limited to a fixed value to ensure that the frequency pulse signal with a certain range and the corresponding relation with pressure is output.

Compared with the traditional bellows pressure altimeter, the vibration cylinder pressure altimeter is with 
the advantages of high precision, simple structure, small volume and light quality.

\subsection{Silicon resonant pressure altimeter}

The silicon resonant pressure sensor consists of two parts. One part is the pressure membrane and the other part is the oscillator. The two parts are closely combined and about 2 microns is only kept in the middle to make the oscillator produce the movement. The pressure membrane is connected with the measured air source at work and the resonant beam is installed on the sensor. There is a pressure difference between the measured air source and the pressure membrane. The pressure membrane deformation is made by the pressure difference, where a deformation force exists. The force is used on the resonant beam leading the natural frequency of resonant beam changed. The natural frequency has the linear function relation with the measured air source in the scope of work pressure. Therefore, the pressure can be got by measuring the sensor frequency.

When being working, the resonant beam frequency is got by adding the vibration signals on resonant beam to make it produce the vibration. The sensor resonant beam is shown in Figure 5.

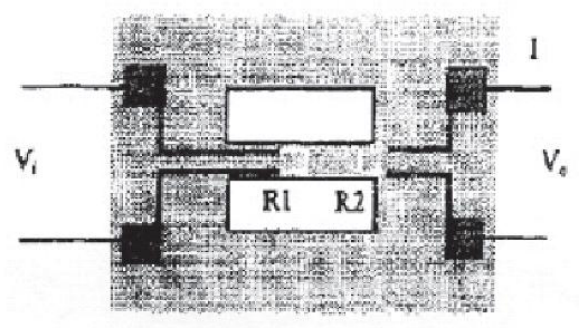

Figure 5.Structure diagram of resonant beam

Two resistances are respectively put on the silicon beam and the resistance in the middle of silicon beam is the vibration resistance. The thermal excitation voltage $V_{1}$ uses the constant-current source for power and the vibration pick-up signal is $V_{0}$. The sensor structure is shown in Figure6.

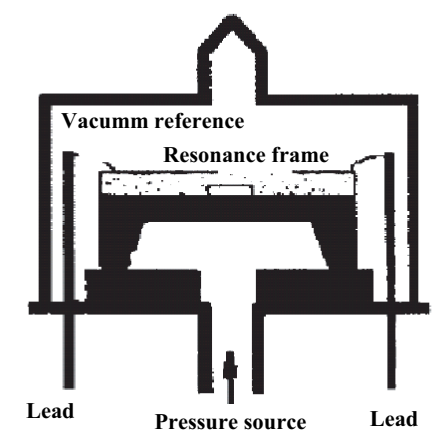

Figure 6.Structure diagram of silicon resonant pressure sensor

Due to the silicon resonant pressure sensor advantages of small volume, light weight, high precision and simple output signal processing, it can be got an intelligent pressure test system with the single-chip computer or dedicated computer. The digital signals of total pressure, static pressure and total temperature are sent to the computer directly at the static pressure tube exit and the needed are calculated by the computer. This way can reduce the delay error of hydrostatic pressure, improve the dynamic characteristics of flight parameters, reduce the member of airborne computer and play the computer potential abundantly.

\subsection{Silicon piezoresistive pressure altimeter}

When the pressure is made on a semiconduct along one axial, the semiconduct will produce the deformation and its resistivity will change significantly. And the phenomenon is called the. The sensor made of the piezoresistive effect of semiconduct materials is called the piezoresistive sensor.

The structure of silicon piezoresistive pressure altimeter is shown in Figure7. The system detects the air static pressure through the silicon resistance pressure sensor and completes the sampling for sensor analog signal through the $\sum-\triangle A D C$ converter. The temperature sensors are directly integrated within the pressure sensor, which can insure that the temperature detected by sensor is accordance with the actual temperature of silicon piezoresistive pressure sensor.

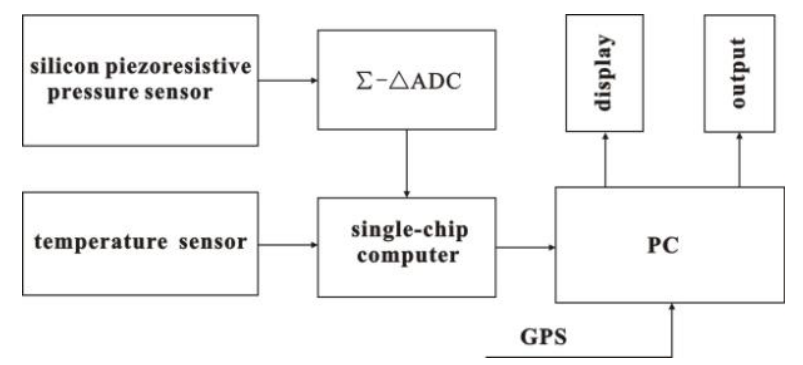

Figure 7.Structure diagram of silicon piezoresistive pressure altimeter

When the aircraft speed is lower than 1 Mach, the aircraft geopotential height is measured indirectly through the air static pressure by the barometric altimeter based on equation (6) in international standard atmosphere environment.

$$
\left\{\begin{array}{l}
H=\frac{T_{b}}{U}\left[\left(\frac{p_{H}}{p_{b}}\right)^{-U_{R / g}}-1\right] \\
H=11000+\left(\frac{R T_{b}}{g}\right) \ln \left(\frac{p_{b}}{p_{H}}\right)
\end{array}\right.
$$

$T_{\mathrm{H}}$ is the atmospheric temperature lower limit value and $P_{\mathrm{H}}$ is the pressure lower limit value of international standard atmosphere in appropriate level.

$U$ is the vertical temperature gradient of international standard atmosphere in appropriate level.

$T_{\mathrm{b}}$ is the standard atmosphere sea-level temperature and $T_{0}=288.15 \mathrm{~K}(0 \sim 11000 \mathrm{~m})$.

$P_{\mathrm{b}}$ is the standard atmosphere sea-level static pressure and $P_{0}=101325 \mathrm{~Pa}$.

$$
\begin{gathered}
R \text { is the air special gas constant and } \\
R=287.05287 m^{2} /\left(K^{\mathrm{o}} \mathrm{s}^{2}\right) \text {. }
\end{gathered}
$$


$\mathrm{g}$ is the gravity acceleration of standard sea level and $g=9.80665 \mathrm{~m} / \mathrm{s}^{2}$.

It should be pointed out that the geopotential height measured by barometric altimeter has the same datum with the altitude (geometry) but two data are not consistent. With the height increasing, the difference is more and more. The conversion can be made according the following equation (7). In the equation, $r$ is the earth radius.

$$
H=r H /(r+h)
$$

The silicon piezoresistive pressure sensor is made of the silicon piezoresistive effect of semiconductor material. The silicon piezoresistive pressure altimeter has the characteristics of high sensitivity, quick dynamic response, high measure precision, good stability, wide operating temperature range, miniaturization, mass production. But the silicon piezoresistive pressure sensor outputs are susceptible to a variety of factors, such as temperature, humidity and power fluctuations. And the cross sensitivity of pressure and temperature is the main influencing factor.

\section{Conclusion}

The pressure measurement technology development is concentrated in two aspects. One is using the micro mechanical processing technology and very nature integrated technology to make the pressure sensor volume as small as possible and to meet the need of special occasions or make the pressure sensor be easy to connect with the computer to develop the intelligent pressure sensor. The second is using the emerging semiconductor materials, such as $\mathrm{SiC}$ and POR-Si or improving the Wheatstone circuit, such as Double Wheatstone bridge circuit and eight arms bridge circuit.

\section{References}

1. Wei Dang, Shengguo Huang, Design of a digital atmospheric pressure altimeter. (Measurement and control technology, 23(12), 2004)

2. Zhenhai Hao, Shengguo Huang, Development of high precision barometric altimeter. (Journal of Nanjing University of Aeronautics \& Astronautics, 41(1), 2009)

3. Limin Chang, Xinglong Qi, Qingwei Dong, Realization of air data computer system based on silicon resonant pressure sensor. (Computer Measurement \& Control, 16(12), 2008)

4. Lei Gao, Yinhui Ma, Wenqing Wang, Design and implementation of silicon piezoresistive barometric altimeter. (Microcomputer \& Applications, 33(20), 2014)

5. Kai Chen, Chaohui Hu, Yuechun Wei, Jie Yan, Design of air data system of an aviation standby instrument system. (Chinese Journal of Sensor and Actuators, 19(4), 2006)

6. Yuhua Wang, Xiaonan Wu, Jinyong Yu, Design of barometric altimeter based on single chip microcomputer. (Instrument Technology, 8, 2013) 